International Mathematical Forum, 2, 2007, no. 61, 3001 - 3012

\title{
Minimal Overlap Rules for Bankruptcy
}

\author{
Ruud Hendrickx, Peter Borm ${ }^{1}$, Roel van Elk and Marieke Quant \\ Center and Department of Econometrics and Operations Research \\ Tilburg University
}

\begin{abstract}
This paper introduces a new way of representing bankruptcy rules. These representations are used to show that the minimal overlap rule is a composition of the Ibn Ezra rule and the constrained equal losses rule, a result originally obtained by [1]. The residual minimal overlap rule is analysed as an alternative extension of the Ibn Ezra rule, by using a composition with the constrained equal awards rule.
\end{abstract}

Mathematics Subject Classification: $91 \mathrm{~A} 12$

Keywords: bankruptcy, representations, Ibn Ezra rule, minimal overlap rule, residual minimal overlap rule, duality

\section{Introduction}

The seminal paper [4] on bankruptcy problems starts with a discussion on a solution proposed by the Talmudic scholar Ibn Ezra. This rule has a natural interpretation in terms of players putting their claims on specific parts of the available estate. One drawback of this rule, however, is that it is only defined for situations in which the estate does not exceed the largest claim.

[4] extends Ibn Ezra's rule to the class of all bankruptcy situations, the result of which [5] calls the minimal overlap rule. Its definition, though natural, is rather implicit and no explicit formula for this rule exists. Hence, very little is known about this rule.

This paper presents a new look on the Ibn Ezra rule and the minimal overlap rule. We first provide an insightful pictorial way of representing bankruptcy

\footnotetext{
${ }^{1}$ Corresponding author. P.O. Box 90153, 5000 LE Tilburg, The Netherlands. E-mail: pemborm@uvt.nl
} 
rules in terms of partial derivatives with respect to the estate. With the help of these representations, we can decompose the minimal overlap rule into two parts: for an estate smaller than the largest claim, it coincides with the Ibn Ezra rule and for larger estates, it equals the Ibn Ezra rule on part of the estate (the largest claim) plus the constrained equal losses rule on the residual claims and estate. [1] proved the same result algebraically by distinguishing between various cases.

One obvious question arising from this decomposition is what happens if on the residual part we apply the constrained equal awards rule rather than the constrained equal losses rule. We provide an interpretation of this rule, which we call the residual minimal overlap rule, in terms of putting claims on specific parts of the estate. This interpretation turns out to have a dual flavour to the idea behind minimal overlap.

The remainder of this paper is organised as follows. In Section 2, we consider alternative representations of bankruptcy rules. In Section 3, we consider the minimal overlap rule and show that it can be decomposed into an Ibn Ezra part and a constrained equal losses part. The residual minimal overlap rule is the topic of Section 4.

\section{Bankruptcy rules and representations}

A bankruptcy situation (cf. [4]) is a triple $(N, E, c)$, where $N=\{1, \ldots, n\}$ is the set of players, $E \geq 0$ is the estate to be divided and $c \in \mathbb{R}_{+}^{N}$ is the vector of claims such that $\sum_{i \in N} c_{i} \geq E$. Throughout the paper we assume that $c_{1} \leq c_{2} \leq \ldots \leq c_{n}$ and the total of claims is denoted by $C=\sum_{i \in N} c_{i}$. The class of bankruptcy situations with player set $N$ is denoted by $B R^{N}$.

A bankruptcy rule is a function $\varphi: B R^{N} \rightarrow \mathbb{R}^{N}$ that assigns to each bankruptcy situation $(N, E, c) \in B R^{N}$ a payoff vector $\varphi(N, E, c) \in \mathbb{R}^{N}$ such that $0 \leq \varphi(N, E, c) \leq c$ (reasonability) and $\sum_{i \in N} \varphi_{i}(N, E, c)=E$ (efficiency). In this paper, we only study bankruptcy rules for fixed $N$.

We further assume that bankruptcy rules are continuous and componentwise (weakly) increasing in $E$, which holds for almost all rules that are known in the literature. We study the shapes of the payoffs $\varphi_{i}$ as function of $E$, for a fixed claim vector $c$. A representation of $\varphi$ is a vector of functions $f^{\varphi, c}=\left(f_{i}^{\varphi, c}\right)_{i \in N}$, where for all $i \in N, f_{i}^{\varphi, c}:[0, C] \rightarrow \mathbb{R}$ is such that 


$$
\varphi_{i}(N, E, c)=\int_{0}^{E} f_{i}^{\varphi, c}(x) d x
$$

for all $E \in[0, C]$. Given our assumptions, $\varphi_{i}$ is differentiable almost everywhere (a.e.). Therefore, a representation always exists. Furthermore, it is uniquely defined and nonnegative a.e. Also, because $\varphi$ is efficient, we have that for all $x \in[0, C], \sum_{i \in N} f_{i}^{\varphi, c}(x)=1$ a.e.

The proportional rule is defined by $P R O P_{i}(N, E, c)=\frac{c_{i}}{C} E$ for all $i \in N$. A representation for $n=3$ is depicted in Figure 1 . For each $i \in N, f_{i}^{P R O P, c}$ is a constant function. In the graph, we indicate for each of the three functions the corresponding player.

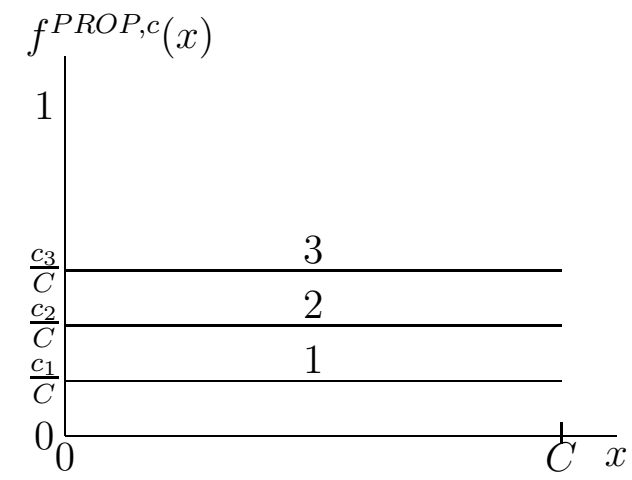

Figure 1: $f^{P R O P, c}$

Before showing representations of some other bankruptcy rules, we first consider duality. The dual $\bar{\varphi}$ of a bankruptcy rule $\varphi$ (cf. [2]) is defined by $\bar{\varphi}(N, E, c)=c-\varphi(N, C-E, c)$ for all $(N, E, c) \in B R^{N}$. Define for each representation $f^{\varphi, c}$ a dual representation $\bar{f}^{\varphi, c}$ by $\bar{f}_{i}^{\varphi, c}(x)=f_{i}^{\varphi, c}(C-x)$ for all $x \in[0, C]$ and $i \in N$. Note that $\bar{f}^{\varphi, c}$ is obtained from $f^{\varphi, c}$ by mirroring in the line $x=\frac{1}{2} C$.

Proposition 2.1 If $f^{\varphi, c}$ is a representation for $\varphi$, then $\bar{f}^{\varphi, c}$ is a representation for $\bar{\varphi}$.

Proof: Let $\varphi$ be a bankruptcy rule, let $c$ be a claims vector and let $f^{\varphi, c}$ be a representation of $\varphi$. Then for all $i \in N$,

$$
\int_{0}^{E} \bar{f}_{i}^{\varphi, c}(x) d x=\int_{C-E}^{C} f_{i}^{\varphi, c}(x) d x
$$




$$
\begin{aligned}
& =\int_{0}^{C} f_{i}^{\varphi, c}(x) d x-\int_{0}^{C-E} f_{i}^{\varphi, c}(x) d x \\
& =\varphi_{i}(N, C, c)-\varphi_{i}(N, C-E, c) \\
& =c_{i}-\varphi_{i}(N, C-E, c) \\
& =\bar{\varphi}_{i}(N, E, c) .
\end{aligned}
$$

As a result of the previous proposition, a bankruptcy rule $\varphi$ is self-dual $(\varphi=\bar{\varphi})$ if and only if $f^{\varphi, c}=\bar{f}^{\varphi, c}$ a.e. for every claims vector $c$.

The constrained equal awards rule is defined by $C E A_{i}(N, E, c)=$ $\min \left\{c_{i}, \alpha\right\}$, where $\alpha$ is such that $\sum_{i \in N} C E A_{i}(N, E, c)=E$. Its dual is the constrained equal losses rule, defined by $C E L_{i}(N, E, c)=\max \left\{c_{i}-\beta, 0\right\}$, where $\beta$ is again determined by efficiency. Representations of these dual rules are depicted for $n=4$ in Figure 2. Again, we indicate on each line segment the players involved. Where a player is absent, the value of his representation function is 0 . We abbreviate $d_{1}=c_{1}+c_{2}+2 c_{3}$.

Note that in view of Proposition 2.1, the representations of $C E A$ and $C E L$ are each other's mirror image.
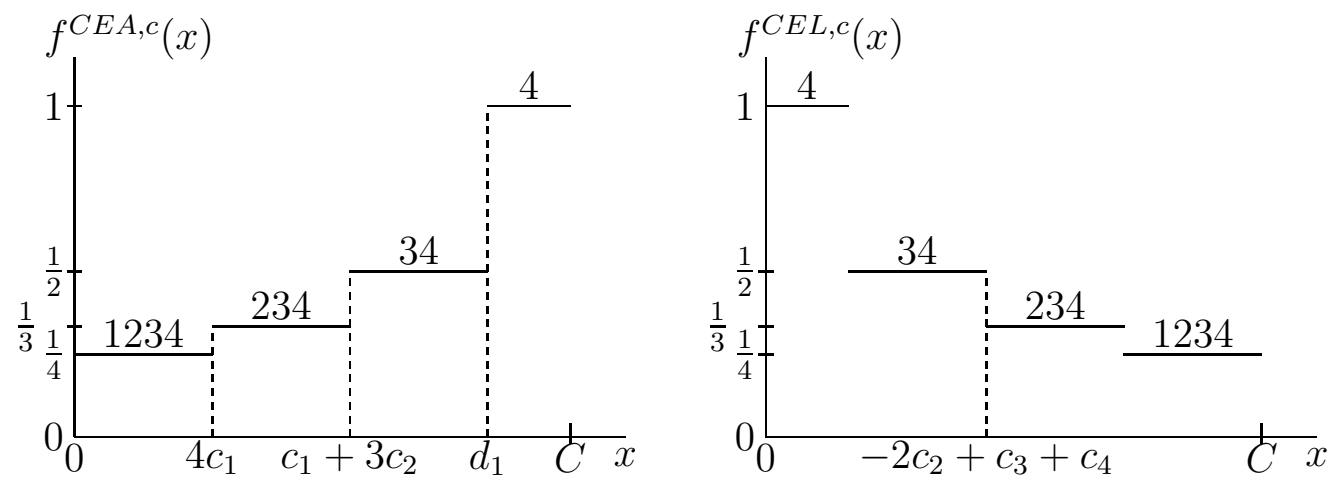

Figure 2: $f^{C E A, c}$ and $f^{C E L, c}$

The Talmud rule (cf. [2]) is defined by

$$
T A L(N, E, c)= \begin{cases}C E A\left(N, E, \frac{1}{2} c\right) & \text { if } E \geq \frac{1}{2} C, \\ c-C E A\left(N, C-E, \frac{1}{2} c\right) & \text { if } E<\frac{1}{2} C .\end{cases}
$$

This rule is self-dual and combines the ideas of constrained equal awards and 
constrained equal losses. A representation for $n=4$ is depicted in Figure 3. Note that since $T A L$ is self-dual, the graph is symmetric in the line $x=\frac{1}{2} C{ }^{2}$

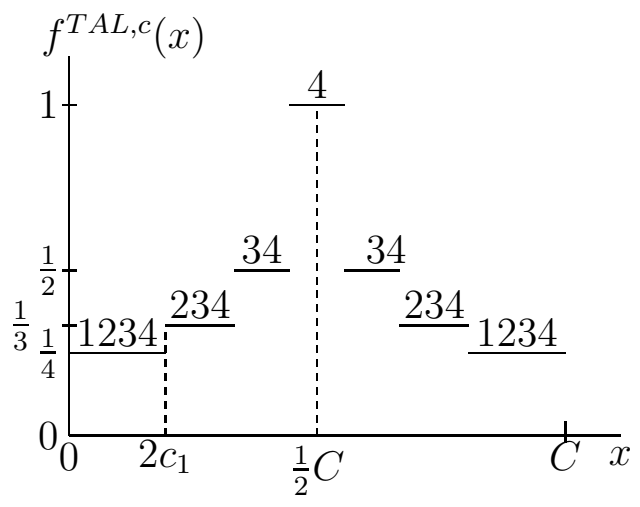

Figure 3: $f^{T A L, c}$

\section{The minimal overlap rule}

The first bankruptcy rule mentioned by [4] is Ibn Ezra's solution, named after a Talmudic scholar from the 12 th century. This rule, $I E$, is only defined for $E \leq c_{n}$ and draws on the idea that each player has a claim on a specific part of the estate. O'Neill uses the example with $E=120$ and $c=(30,40,60,120)$. Player 1 claims 30, but this 30 is claimed by all four players, so player 1 should receive only a quarter of his claim. Player 2 should receive his part of the 30 plus a third of his residual claim of 10, and so on.

Ibn Ezra's method is illustrated in Figure 4. Each player has a claim on a specific part of the estate, represented by line segments, and these claims are nested. Each part of the estate is divided equally among the players having a claim on it. The outcome for the example in Figure 4 is $\left(7 \frac{1}{2}, 10 \frac{5}{6}, 20 \frac{5}{6}, 80 \frac{5}{6}\right)$.

Because Ibn Ezra's method can only be applied if the estate does not exceed the largest claim (otherwise part of the estate would remain unclaimed), a representation only exists on $\left[0, c_{4}\right]$. Claims that are larger than the estate are simply truncated so that they fit exactly within the estate. Figure 5 depicts a representation of the $I E$ rule.

There are of course various ways to extend the $I E$ rule to the full domain of estates. [4] presents one natural extension, which is based on a property he

\footnotetext{
${ }^{2}$ From the examples in this section, one might think that all well-known bankruptcy rules are piecewise linear in the estate. [3], however, shows that the adjusted proportional rule is not. As a consequence, a representation of this rule is not a step function.
} 


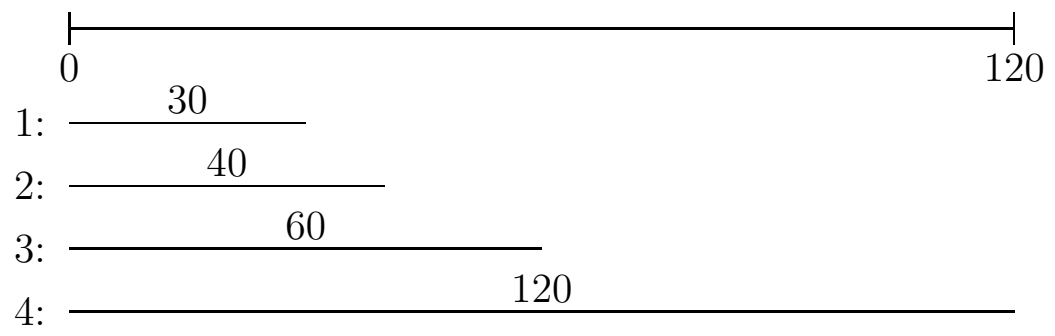

Figure 4: $\operatorname{IE}(N, 120,(30,40,60,120))$

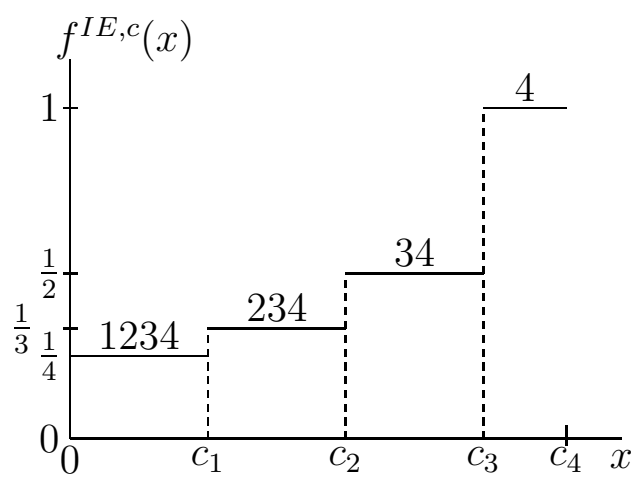

Figure 5: $f^{I E, c}$

calls "lexicographic minimisation of conflict by extent". The players' claims (again, truncated to the estate) are arranged such that the size of the part of the estate claimed by exactly one player is maximal, and given this, the size of the part of the estate claimed by two players is maximal, and so on. Obviously, if $E$ does not exceed the largest claim, then this boils down to Ibn Ezra's method. O'Neill names the resulting rule, which he proves to be uniquely determined by this property, the extended Ibn Ezra rule. We follow [5] in calling it the minimal overlap rule, $M O$.

As a consequence of lexicographic minimisation of conflict by extent, the first $t$ units of each claim are overlapping, while the residual claims are put disjointly on the estate. So, to compute the $M O$ solution, which will be illustrated in the next example, we have to find a $t$ such that

$$
g(t)=E,
$$

where $g: \mathbb{R} \rightarrow \mathbb{R}$ is defined by

$$
g(t)=\sum_{i \in N}\left(c_{i}-t\right)_{+}+t
$$


for all $t \in \mathbb{R}$. The solution of (3.1) is unique, except when $c_{n-1}<E \leq c_{n}$, in which case we take $t=E$.

Example 3.1 Consider $N=\{1, \ldots, 4\}, E=10$ and $c=(2,3,6,8)$. Then $t=4$. The arrangement of the claims is depicted in Figure 6 .

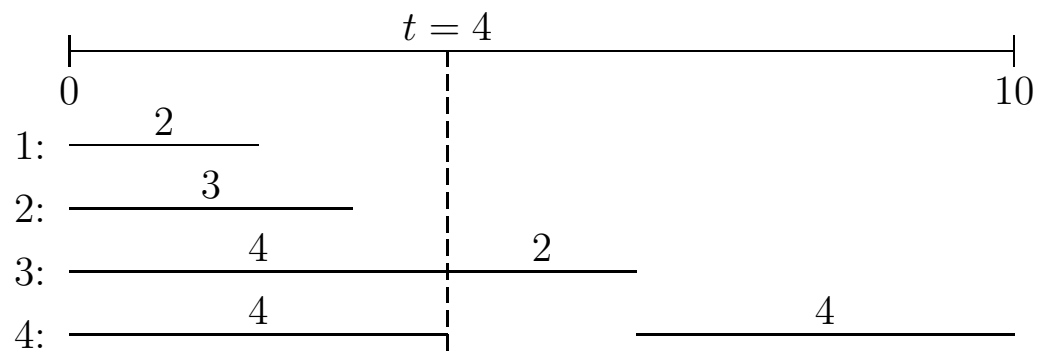

Figure 6: $\operatorname{MO}(N, 10,(2,3,6,8))$

The payoff to player 1 equals $\frac{1}{2}$, player 2 gets $\frac{1}{2}+\frac{1}{3}=\frac{5}{6}$, player 3 receives $\frac{5}{6}+\frac{1}{2}+2=3 \frac{1}{3}$ and the remaining $5 \frac{1}{3}$ is for player 4 .

In order to give a representation for $M O$, we need to know more about the $t$ defined by (3.1). If $E \leq c_{n}$, then it follows immediately from claims truncation that $t=E$. For $E>c_{n}, t$ is computed by applying the inverse function of $g$ to $E$. For four players, the piecewise linear function $g$ is depicted in Figure 7, as well as the solution of (3.1). For brevity, $d_{1}=-c_{2}+c_{3}+c_{4}$ and $d_{2}=-2 c_{1}+c_{2}+c_{3}+c_{4}$.

Using the construction of $t$ in Figure 7, we can now find a representation of the $M O$ rule. If the estate does not exceed the largest claim, the $M O$ rule coincides with the $I E$ rule and hence, their representations coincide. If $E$ increases from $c_{4}$ to $d_{1}$, then $t$ goes down from $c_{3}$ to $c_{2}$. So on this interval, the overlapping parts of the claims of players 3 and 4 decrease (at the same speed), while for the other players nothing changes. Hence, $f_{3}^{M O, c}(x)=f_{4}^{M O, c}(x)=\frac{1}{2}$ for all $x \in\left[c_{4}, d_{1}\right]$. Similarly, players 2,3 and 4 receive an equal share when the estate increases from $d_{1}$ to $d_{2}$, while on $\left[d_{2}, C\right]$, a rising estate benefits all players. Comparing the representation of the $M O$ rule with those of the $I E$ rule and $C E L$ rule, we arrive at the following theorem, which was originally proved by [1].

Theorem 3.1 Let $(N, E, c) \in B R^{N}$. Then 


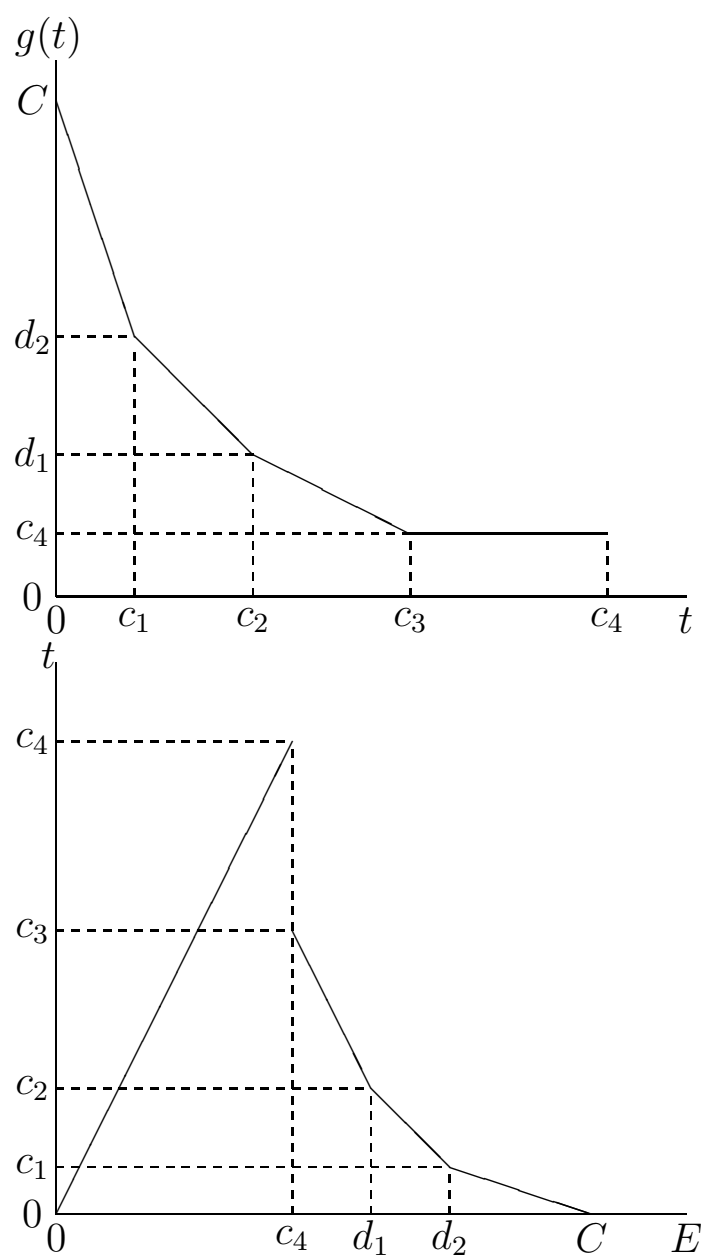

Figure 7: Determining $t$ for $M O$

$$
M O(N, E, c)= \begin{cases}I E(N, E, c) & \text { if } E \leq c_{n}, \\ I E\left(N, c_{n}, c\right)+C E L\left(N, E-c_{n}, c^{\prime}\right) & \text { if } E>c_{n},\end{cases}
$$

where $c^{\prime}=c-I E\left(N, c_{n}, c\right)$.

Proof: If $E \leq c_{n}$, then the assertion immediately follows from the construction of the $M O$ rule. If $E>c_{n}$, then $I E_{n}\left(N, c_{n}, c\right)-I E_{n-1}\left(N, c_{n}, c\right)=c_{n}-c_{n-1}$. Hence, $c_{n}^{\prime}=c_{n-1}^{\prime}$. (Note also that $c_{1}^{\prime} \leq c_{2}^{\prime} \leq \ldots \leq c_{n-1}^{\prime}$.) Because the two largest residual claims are equal, applying $C E L$ to $\left(N, E-c_{n}, c^{\prime}\right)$ results in the representation of Figure 2, without the separate segment for player $n$. So, $f^{M O, c^{\prime}}$ on $\left[c_{n}, C\right]$ boils down to $f^{C E L, c^{\prime}}$ on $\left[0, C-c_{n}\right]$. From this, the statement readily follows. 


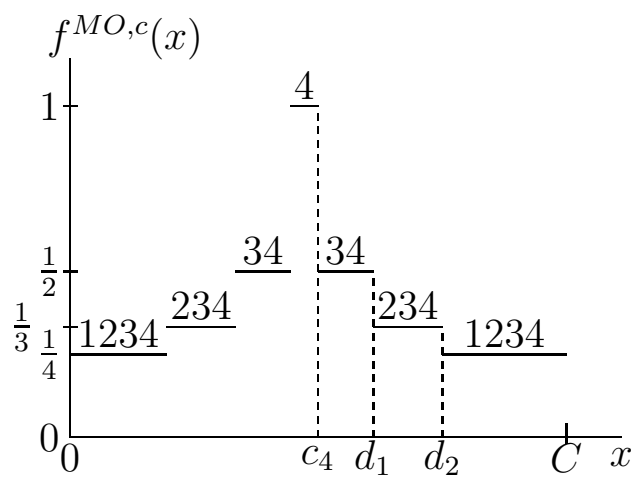

Figure 8: $f^{M O, c}$

\section{The residual minimal overlap rule}

One natural question arising from Theorem 3.1 is what happens if we use the $C E A$ rule rather than the $C E L$ rule to extend the $I E$ rule. Put differently, how can we interpret the rule whose representation is given in Figure 9, and which for reasons that will become clear later on we call the residual minimal overlap rule $(R M O)$, in terms of putting claims on specific parts of the estate?

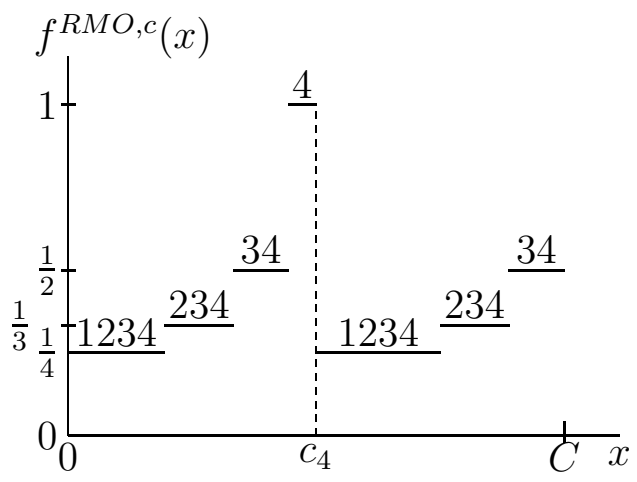

Figure 9: $f^{R M O, c}$

We first provide a definition of the $R M O$ rule ${ }^{3}$ and later on we show that it indeed has a representation as depicted in Figure 9.

For $M O$, the claims are put in such a way as to minimise total overlap, while putting the smallest claims in this overlapping part. For $R M O$, we also minimise total minimal overlap, but now the smallest claims are not situated in the overlapping part. Rather, the disjoint part of the claim has the same

\footnotetext{
${ }^{3}$ This rule is a special case of a family of rules studied in the mimeo "Ibn Ezra and the contested garment princple" by J. Alcalde.
} 
size for all players (with of course the restriction that this should not exceed the claim itself), while the residual claims overlap.

Taking $t$ to be the (maximal) size of the disjoint part of a claim, the size of the overlapping part equals $c_{n}-t$. Hence, we have to find a $t$ such that

$$
h(t)=E,
$$

where $h: \mathbb{R} \rightarrow \mathbb{R}$ is defined by

$$
h(t)=\sum_{i \in N} \min \left\{t, c_{i}\right\}+c_{n}-t
$$

for all $t \in \mathbb{R}$. This $t$ is unique except when $E=C$, in which case we take $t=c_{n-1}$.

Example 4.1 Consider $N=\{1, \ldots, 4\}, E=15$ and $c=(2,3,6,8)$. Then $t=2 \frac{1}{2}$. The arrangement of the claims is depicted in Figure 10 .

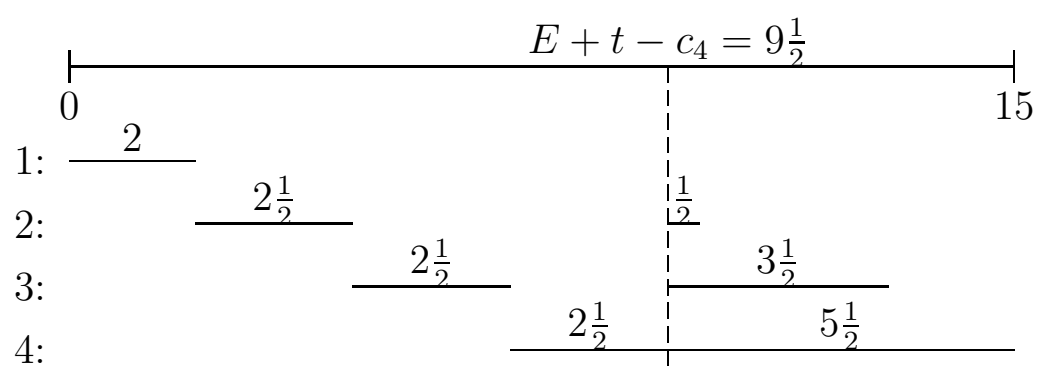

Figure 10: $R M O(N, 15,(2,3,6,8))$

The payoff to player 1 equals 2 , player 2 gets $2 \frac{1}{2}+\frac{1}{6}=2 \frac{2}{3}$, player 3 receives $2 \frac{2}{3}+1 \frac{1}{2}=4 \frac{1}{6}$ and the remaining $6 \frac{1}{6}$ is for player 4 .

Note that for the bankruptcy situation $(N, E, c)$ described in Example 3.1, we have $t=\frac{2}{3}$ and $\operatorname{RMO}(N, E, c)=\left(1,1 \frac{1}{3}, 2 \frac{5}{6}, 4 \frac{5}{6}\right)$.

Again, it is readly seen that if the estate is smaller than the largest claim, the $R M O$ solution and the $I E$ solution coincide.

Figure 11 depicts the graph of $h(t)$, as well as the solution of (4.1). For $E \leq c_{4}$, it follows from claims truncation that $t=0$ is the solution of (4.1). For $c_{4}<E<C$, the solution is given by the inverse function of $h$, while for 


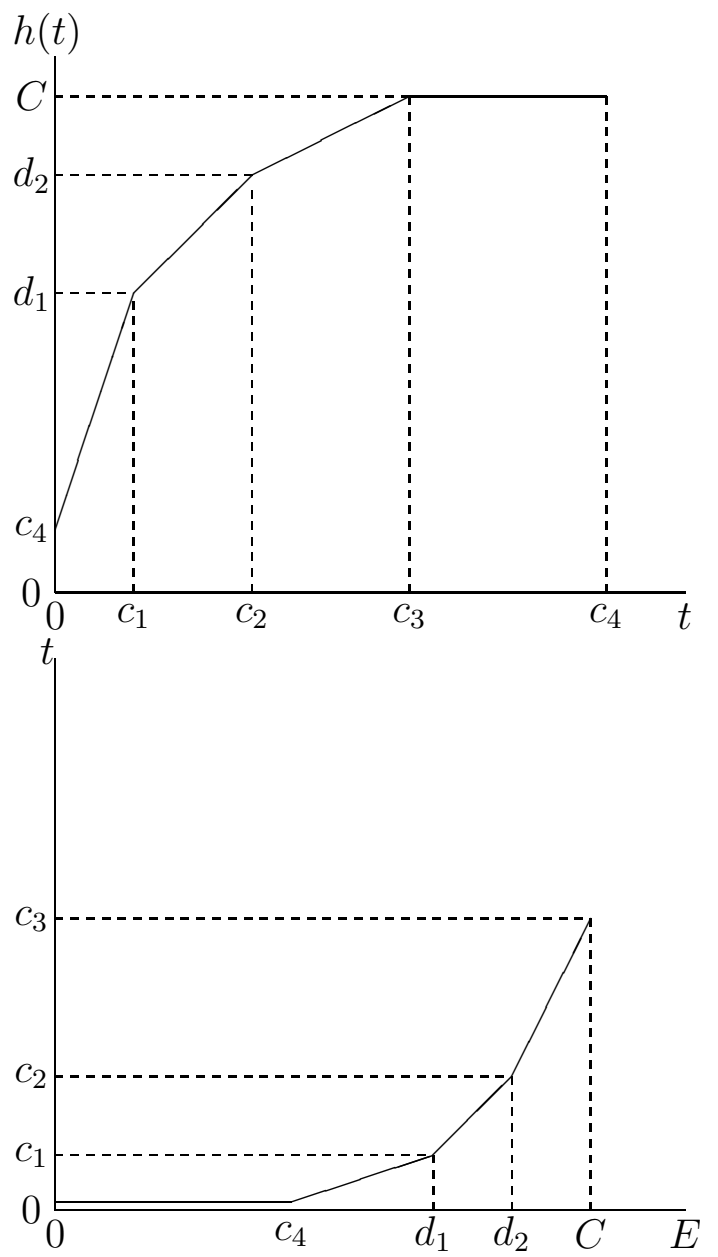

Figure 11: Determining $t$ for $R M O$

$E=C, t=c_{3}$ is taken as the solution. We abbreviate $d_{1}=3 c_{1}+c_{4}$ and $d_{2}=c_{1}+2 c_{2}+c_{4}$.

Obviously, $f^{R M O, c}$ and $f^{I E, c}$ coincide on [0, $\left.c_{4}\right]$. If $E$ goes from $c_{4}$ to $d_{1}$, $t$ increases from 0 to $c_{1}$, so on this interval, all players benefit equally and $f_{i}^{R M O, c}(x)=\frac{1}{4}$ for all $i \in N$ and $x \in\left[c_{4}, d_{1}\right]$. The remainder of the representation in Figure 9 follows similarly.

The following theorem follows in the same way as Theorem 3.1.

Theorem 4.1 Let $(N, E, c) \in B R^{N}$. Then

$$
R M O(N, E, c)= \begin{cases}I E(N, E, c) & \text { if } E \leq c_{n} \\ I E\left(N, c_{n}, c\right)+C E A\left(N, E-c_{n}, c^{\prime}\right) & \text { if } E>c_{n}\end{cases}
$$


where $c^{\prime}=c-I E\left(N, c_{n}, c\right)$.

\section{References}

[1] J. Alcalde, M. del Carmen Marco, J. Silva, The minimal overlap rule revisited, Social Choice and Welfare (to appear).

[2] R. Aumann, M. Maschler, Game theoretic analysis of a bankruptcy problem from the Talmud, Journal of Economic Theory, 36 (1985), 195213.

[3] I. Curiel, Cooperative Game Theary and Applications, Ph. D. Thesis, Nijmegen University, Nijmegen, The Netherlands, 1988.

[4] B. O'Neill, A problem of rights arbitration from the Talmud, Mathematical Social Sciences, 2 (1982), 345-371.

[5] W. Thomson, Axiomatic and game theoretic analysis of bankruptcy and taxation problems: A survey, Mathematical Social Sciences, 45 (2003), 249-297.

Received: June 6, 2007 